\title{
What is LAMPF II?
}

H. A. Thiessen 
DISCLAMER

This report was prepared as in account of work sponsored by an azency of the United States Government. Neither the United States Government nor any agency thereof, nor any of their employees, makes any warranty, express or implied, or assumes any leal liability or responsibility for the accuracy, completeness, or usefulness of any information, apperatus, product, or process dixclosed, or represents that its use would not infringe privately owned rifhts. References herein to any specific commerrial product, proces, or service by trade name, trademerk, manufacturer, or otherwise, does not necesinily conetitute or imply its endorsement, recommendation, or favoring by the United States Government or any aency thereof. The views and opinions of authors exprewad herein do not necesarily state or reflect thow of the United States Government or any agency thereof. 
WHAT IS LAMPF II?

by

H. A. Thiessen

\begin{abstract}
The present conception of LAMPF II is a high-intensity $16-\mathrm{GeV}$ synchrotron injected by the LAMPF $800-\mathrm{MeV} \mathrm{H}{ }^{-}$beam. The proton beam w111 be used to make secondary beams of neutrinos, muons, pions, kaons, antiprotons, and hyperons more intense than those of any existing or proposed accelerator. For example, by taking maximum advantage of a thick target, modern beam optics, and the LAMPF II proton beam, 1t w111 be possible to make a negative muon beam with nearly $100 \%$ duty factor and nearly 100 times the flux of the existing Stopped Muon Channel (SMC). Because the unique features of the proposed machine are most applicable to beams of the same momentum as LAMPF (that $1 \mathrm{~s},<2 \mathrm{GeV} / \mathrm{c}$ ), it may be possible to use most of the experimental areas and some of the auxillary equipment, including spectrometers, with the new accelerator. The complete facility will provide improved technology for many areas of physics already available at LAMPF and will allow expansion of medium-energy physics to include kaons, antiprotons, and hyperons. When LAMPF II comes on line in 1990 LAMPF will have been operational for 18 years and a major upgrade such as this proposal will be reasonable and prudent.
\end{abstract}

I. NUCLEAR PHYSICS AT LAMPF II

The areas of nuclear physics that can be studied with LAMPF II are listed below.

- Hypernuclei - In hypernuclei we will be able to study nuclear structure wth one or more strange quarks in the nuclear bag. 
- Hyperons - By studying the decays of the excited states we can determine the wave functions of the three quarks that make up the hyperons.

- Kaon-Nucleus Scattering - The kaon is the hadron with the longest mean-free path in nuclear matter and as such will be an excellent probe of nuclear struc ture.

- Plon-Nucleus Scattering - we can use 0.5 - to $1.0-\mathrm{GeV}$ plons for nuclear structure studies with the advantage that the mean-free path will be much longer and the selectivity for magnetic transitions will be significantly better than at the Energetic Pion Channel Spectrometer (EPICS).

- Hadron Resonances in Nuclear Matter - Recent work has shown that $\Delta$-hole modes are an important part of nuclear structure. Using kaons will make it possible to excite the $\mathrm{Y}^{*}(1520)$, which has a much narrower width and longer 1ifetime. This should be a much cleaner case to study than the $\Delta$.

- Muon Capture and Muon Spin Rotation ( $\mu S R$ ) - The high flux of muons from LAMPF II will make possible significantly more sensitive experiments in mion capture, which studies the weak interaction in nuclear matter at a momentum transfer comparable to the muon mass, and in muon spin rotation, which is useful for solid-state applications.

- Neutrino-Nucleus Scattering - By using the high flux of neutrinos from LAMPF II we can study the spin and isospin structure of the charged current by scattering from selected light nucle1.

- Exotic Atoms - In addition to $\mathrm{k}^{-}, \bar{\Sigma}$, and $\mathrm{p}^{-}$atoms that have already been studied at existing machines, $1 t$ will be possible to use intense beams of kaons and antfprotons to make $\Xi^{-}, \Omega^{-}$, and $\bar{\Sigma}^{-}$atoms that have not yet been seen. By studying the atomic spectra we can determine masses, magnetic moments, and the low-energy baryon-nucleus potentlal for these rare particles.

II. PARTICLE PHYSICS AT LAMPF II

The classes of particle physics that w111 be studied at LAMPF II are 11sted below.

- Rare Kaon Decays - There are many rare-decay modes that provide extremely sensitive tests of the standard Weinberg-Salam-Glashow gauge model of the electromagnetic and weak interactions. Other decay modes are sensitive to proposed extensions of this model. In many cases the measurements of the 
rare-decay modes possible with LAMPF II are more sensitive than any other experiment planned at any existing or proposed accelerator.

- Charge-Parity (CP) Violation in Kaon Decays - The decay of the kaon is the only verified example of violation of CP symmetry known. The origin of this CP violation has important consequences for unification. Our knowledge of all the observables of CP violation is presently limited by statistical uncertainties; hence high-intensity beams from LAMPF II will have a major impact on this field.

- Neutrino Oscillations and Neutrino-Electron Scattering - The high flux of neutrinos of variable energy from LAMPF II w111 make possible neutrino mass searches with 100 times the sensitivity of those performed at existing accelerators. It will also be possible to study neutrino- and antineutrinoelectron scattering with sufficient precision and statistics to determine the angular distribution. Such precise experiments are important tests of the standard theory of electroweak interactions and its possible extensions.

- Muonium - The intense beam of muons from LAMPF II will make possible the study of muonium with precise tests of quantum electrodynamics at a level where weak-interaction effects become visible.

- Pion, Kaon, and Hyperon-Nucleon Scattering - Although this field has been studied in the past, many important puzzles remain. In particular, there are no spin-rotation experiments; the kaon-nucleon polarization is poorly known, especially at low energy; and the hyperon-nucleon problem is practically untouched. Much work remains to be done to clean up hadron spectroscopy.

- Antiproton Physics - It will be difficult to compete with the high-quality cooled beam of antiprotons from the low-energy antiproton ring (LEAR) at CERN. However, possibilities exist for much higher intensity beams of antiprotons at LAMPF II. An important experiment that will make use of a high-intensity, high-momentum beam is $\bar{p}+p+e^{+}+e^{-}$, which wili measure the proton form factor in the time-like region inaccessible in electron scattering. 


\section{LAMPF II ACCELERATOR}

The basic machine for LAMPF II is a rapid-cycling $16-\mathrm{GeV}$ synchrotron. The high flux will be obtained by operating at $60 \mathrm{~Hz}, 100$ times faster than existing machines. With $10^{13}$ protons per pulse, which has already been achieved at many laboratories, we w111 have an average current of $100 \mu \mathrm{A}$. The major technical problems to be solved are the large amount of $r f$ power required and the minimizing of beam losses during the acceleration cycle.

To achieve a good duty factor the rapid cycler will inject its beam into a dc-stretcher ring. Slow extraction, with nearly $100 \%$ duty factor, will be done from the stretcher ring. We also require good rf timing capability of better than $1-n s$ pulse width at $50 \mathrm{MHz}$ or lower repetition rate. This good timing will make possible particle identification without detectors in the beam and may also make possible rf separated beams. Providing both high intensity and good timing capability simultaneously is a difficult but probably manageable task for our accelerator designers.

No decision has been made on the sharing of the primary beam from LAMPF II. For planning purposes we assume there will be four primary beams, each receiving an average of $25 \mu \mathrm{A}$. One of the four beams will be a fast-extracted variableenergy beam dedicated to neutrino physics; the remaining three beams will be slow-extracted beams, which will be used to produce secondary beams. We assume that there will be three thick targets, each of more than one interaction length.

\section{LAMPF II EXPERIMENTAL AREAS}

It may be possible to locate the accelerator on the site in such a way that the existing experimental areas can be provided with $16-\mathrm{GeV}$ proton beams. A site layout that meets this requirement is shown in Fig. 1. The accelerator would be located in a tunnel $\sim 9.14 \mathrm{~m}$ below the present beam elevation. We are also considering constructing completely new experimental areas, but this plan is less advanced.

A possible plan for the experimental areas is shown in Fig. 2. It is clear from such layouts that the LAMPF II experimental areas will be comparable in scope to those already in use at LAMPF. The layout shows all the presently envisaged beam lines except the neutrino line, which will be a short line from one of the straight sections of the accelerator. Of course this is only a preIminary sketch and is subject to considerable change. 


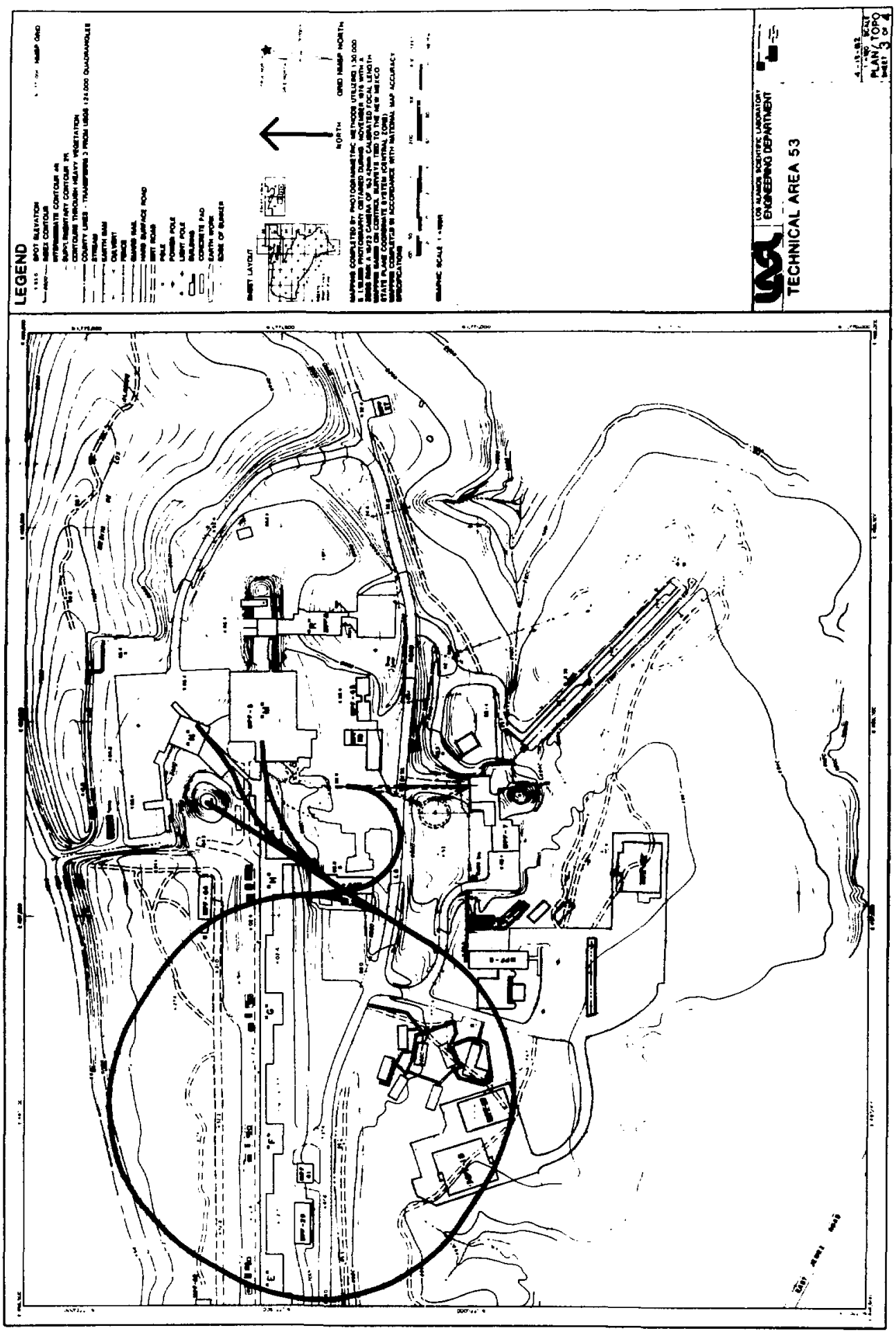

Fig. 1 .

Proposed site layout for LAMPF II. 


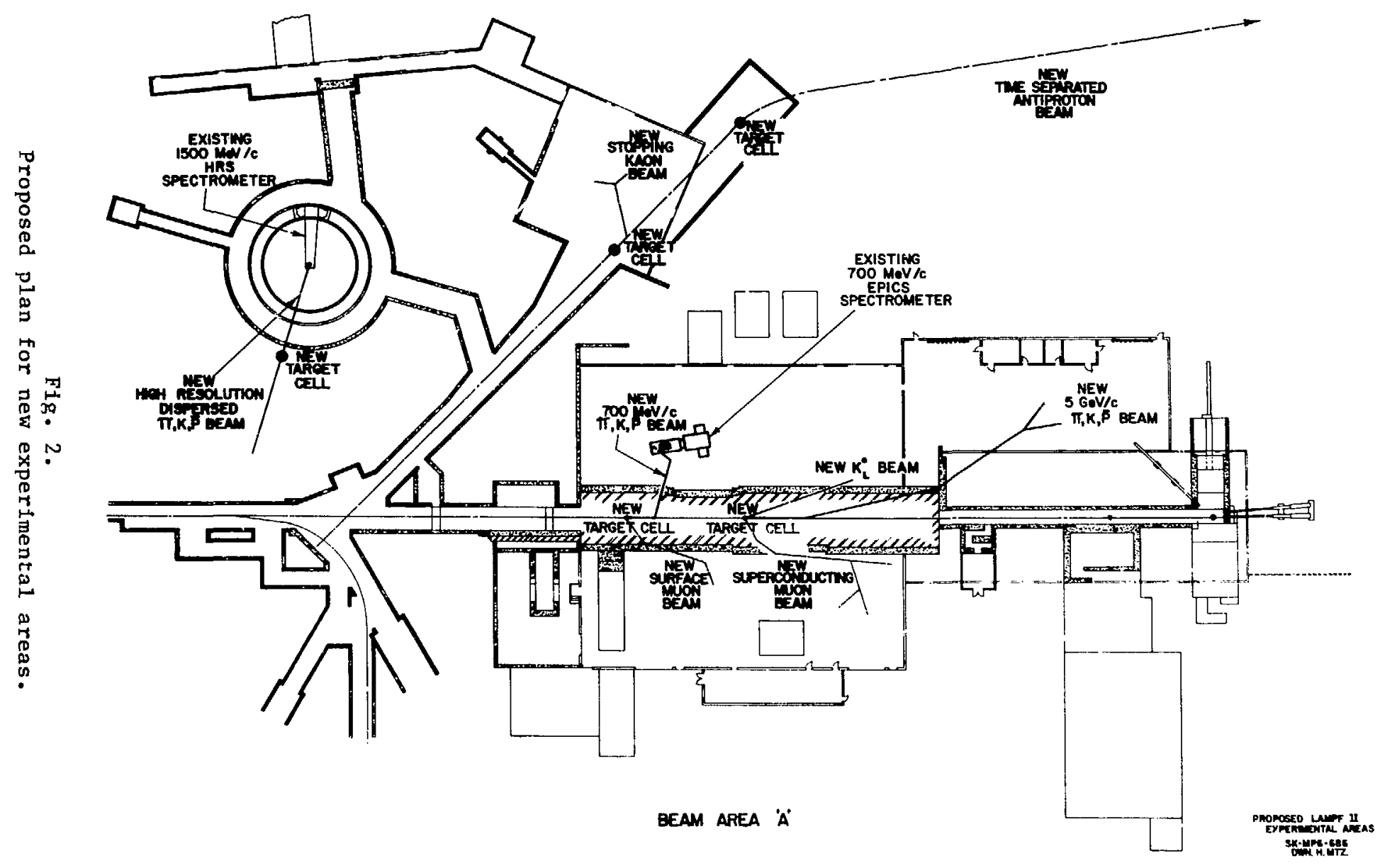


V. EXTENSIONS TO THE BASIC PLAN

We are actively pursuing two directions in addition to the plans mentioned above. First, we are looking at the possibility of providing a cooled antiproton beam such as LFAR at CERN. It will be difficult to provide a competitive proposal unless we come up with a new-idea antiproton beam. The NucleonAntinucleon Working Group is studying this problem.

A second possibility is that we construct a colliding-beam facility for polarized protons and heavy ions. Such a facility would provide a unique capabil1ty if we can achieve adequate luminosity. If the interference with the operation of the other facilities can be kept to a few hours a day we would consider this a feasible option. Nick Diglacomo of $P$ Dirision is looking at the feasibility of a collider facility.

VI. COST AND TIME SCALE

We hope to complete a plan for the accelerator and experimental areas during 1983; a proposal could be ready for submission at the end of 1983 . If approved, construction could start in FY 1986 and operation could occur in 1990. A detailed cost estimate rust awat completion of our plan; however, early estimates of $\$ 75 \mathrm{M}$ for the accelerator and $\$ 75 \mathrm{M}$ for experimental areas have been confirmed by several consultants.

VII. USER INPUT FOR LAMPF II

Several working groups of prospective LAMPF II users have been organized. Those presently active are as follows.

1. Nuclear Physics

2. Muons

3. Rare Kaon Decays

4. CP Violation

5. Hyperons

6. Nucleon/Antinucleon

7. Nuclear Chemistry

8. Neutrinos

Each working group wll hold several meetings during the next year to select the most interesting few experiments in each experiment area and to study each experiment carefully enough to demonstrate its feasibility. The 11st of working groups is arbitrary -- changes can be made if destred. The next meetings of the 
working groups will be paraliel sessions at the LAMPF II Workshop, July 19-22, 1982.

VIII. NUCLEAR PHYSICS WORKING GROUP

At the February meeting Carl Dover presented a review of kaon-nucleus physics, Lee Roberts reviewed kaonic atoms, and Jim Carr discussed his first results on the pion-nucleus effective interaction. The pion-nucleus scattering calculations were the most important new results shown at this session and indicated that significant sensitivity to neutron or proton particie-hole states is possible, with magnetic transitions enhanced near $500 \mathrm{MeV}$ and natural-parity transitions dominating near $1000 \mathrm{MeV}$. H. A. Thiessen reviewed the proposed $700-\mathrm{MeV} / \mathrm{C}$ dispersed kaon beam for use with the EPICS spectrometer, Harald Enge presented his proposal for a low-energy kaon beam separated by an absorber, a: ad Donald Lobb showed his design for an achromatic stopping pion beam proposed for the TRIUMF kaon factory.

At a second meeting held in March, Leonard Pisslinger discussed the University of Washington model for short-range effects in hadron-nucleus scattering based on a quark model, Ben Gibson reviewed the field of low-mass hypernuclei, and Peter Mulders presented a discussion of quark-model predictions for hadron spectroscopy. On the experimental side $H$. A. Thiessen presented first results for a high-momentum dispersed kaon bean proposed for the High-Resolution Spectrometer (HRS) and Ed Hungerford began a discussion of the experimental problems of observing cascade hypernuclei. Morgan May reviewed the earlier Thiessen proposal for a low-momentum kaon beam and, using a good deal of experience from Brookhaven, concluled that this proposal is practical.

The problem facing this working grcup is to narrow the range of experiments to the point where they can be accomplished with a small number (say, two) of beam lines. The problem of separators for a high-momentum beam is still under discussion, as is the question of the origin of backgrounds in a separated beam. The issue of coincidence experiments has not yet been addressed.

IX. N AND $\overline{\mathrm{N}}$ ACTIVITIES

Can we make an antiproton beam that would compete with LEAR? LAMPF II could produce more antiprotons than CERN (especially if we had $32-\mathrm{GeV}$ protons instead of $16 \mathrm{GeV}$ ), but could we cool them? CERN and Fermi National Accelerator Laboratory (FNAL) are probably close to the limit of stochastic cooling (10ll 
$\overline{\mathrm{p}}$ /day), but Peter McIntyre (Texas A\&M University) and Billy Bonner (Los Alamos) are investigating possible electron-cooling schemes to cool $10^{12} \bar{p} / d a y$.

A good beam of polarized antiprotons would give LAMPF II a major advantage, but how do we do this? Our polarized proton beam is of no help unless the spintransfer coefficients are large. We will measure them, but they are expected to be small. LEAR plans to make antiprotons and then polarize them by scattering, but this destroys the cooled-beam quality. rerhaps we can scatter first and then cool.

If ali grand schemes prove impractical, however, there is still valuable work that we could do with a conventional (uncooled) $\overline{\mathrm{p}}$ secondary beam 1 ine.

crlliding beams of polarized protons would be exciting $(16+16 \mathrm{GeV} \approx$ $500 \mathrm{GeV}$ on a fixed target). The technology is well understood, but if we are to have variable energy without interfering with kaon production we would need two additional rings.

Finally, we could turn our existing polarization expertise to $\mathrm{mp}$, $\mathrm{Kp}$, and $\Lambda_{\mathrm{p}}$ experiments. These fields have been discussed by Kelley and by Cutkosky (the Isgur-Karl catastrophe) in the proceedings of the $31-C e V$ workshop (Los Alamos National Laboratory report LA-8775-C (March 1981), pp. 166 and 185].

X. RARE KAON DECAY WORKING GROUP SUMMARY

Some of the rare decays considered are discussed below.

The standard Weinberg-Salam-Glashow model offers no explanation or differentiation among the three generations of-leptons and quarks observed in nature. New interactions, not contifned in this model: have been proposed to link the generations. Rare lepton-flavor-violating decays, such as $\mathrm{K}^{+} \rightarrow \pi^{+} \mu e$ and $\mathrm{K}^{0} \rightarrow \mu e$, test for the presence of these interactions even if the mass of the particle responsible for these interactions is many tera-electron volts. There is no direct way to searcin for such massive objects.

The decay $\mathrm{K}^{+} \rightarrow \pi^{+} \nu \bar{\nu}$ is suppressed by the Glashow-Iliopolous-Malani (GTM) mechanism. The particular interest in this process is that the decay rate is proportional to the number of (light) neutrino types, thus making it possible to determine the number of lepton generations. The high flux available at LAMPF II is necessary to improve the experimental sensitivity.

The decay $K_{L}^{0} \rightarrow K^{ \pm} e^{ \pm} v$ is expected to have a branciiing ratio of $3 \times 10^{-9}$. This decay provides a sensitive test of the conserved vector current (CVC) hypothesis in the presence of a strange quark. CVC, which is a cornerstone of 
al1 present theorles, has never been tested in this domain. Other rare deciys, which have been discussed and are of great interest, are $\mathrm{K}_{\mathrm{L}}^{0} \rightarrow \mu^{+} \mu^{-}, \mathrm{K}^{+}+\pi^{+} e^{+} e^{-}$, $\mathrm{K}^{0}+\ell^{+} \ell^{-} \gamma$, and $\mathrm{K}^{+} \rightarrow \mathrm{e}^{+} \mathrm{v}$.

\section{CP VIOLATION WORKING GROUP SUMMARY}

Since the discovery of CP violation in 1964, many difficult and elegant experiments have been performed to study this phenomenon. However, the most important issue, namely what interaction is the underlying cause of the observed eff: 's, vemains a mystery. CP violation has been observed only in the neutral kaon stem, but more precise experiments are required to distinguish between various theoretical models of the effect.

The uncertainty in the experimental detemination of every CP-violazion parameter is dominated by statistical errors. The large increase in kaon intensity avallable at LAMPF II will be clearly invaluable in inproving these measurements. We could also design $K^{0}$ beams with improved properties (smailer beam spot and lower neutron contamination, for example) that would permit experiments with smaller systematic errors.

The experfments that have been identified as being particularly important are

- a precise measurement of the rate for $\mathrm{K}_{\mathrm{L}}^{0}+\pi^{0} \pi^{0}$,

- a measurement of the $\mu^{+}$polarization transverse to the decay plane in $\mathrm{K}_{\mathrm{L}}^{0}+\pi^{-}{ }_{\mu}^{+} v_{\mu}$ and $\mathrm{K}^{+}+\pi^{0} \mu^{+} \nu_{\mu}$,

- a search for CP-violating effects in the rare-decay modes $\mathrm{K}^{0}+\mathrm{\mu}^{+} \mathrm{\mu}^{-}$and $\mathrm{K}^{0} \rightarrow \mathrm{r}$,

- a search for the CP-violating decay $K_{S}^{0} \rightarrow \pi^{+} \pi^{-} \pi^{0}$, and

- a comparison of the decays $\mathrm{K}^{+} \rightarrow \pi^{+} \pi^{+} \pi^{-}$and $\mathrm{K}^{-}+\pi^{-} \pi^{+} \pi^{-}$.

\section{NEUTRINO WORKING GROUP SUMMARY}

It is fairly straightforward to identify the physics one wants to address with a neutrino facility at LAMPF II. These are

- neutrino oscillation searches with improved sensitivities. In particular, we could search for $\nu_{\mu}$ disappearance and $\nu_{\mu}$ oscillation into $\nu_{e}$ wth sensitivfties not matched by any other facility.

- neutrino-electron elastic scattering. The $\nu_{\mu}$-e and $\nabla_{\mu}$-e elastic scattering are the simplest weak neutral-current interactions that can be studled. Data of unprecedented accuracy could be obtained at LAMPF II that would 
provide the most stringent test of the electroweak theories and the most accurate measurement possible of the Weinberg angle. The Neutrino Working Group is investigating how to minimize backgrounds and systematic errors.

We could also measure $\nu_{e}-e$ and $\nabla_{e}$-e elastic scattering in a neutrino beam derived from $\mathrm{K}_{\mathrm{L}}^{0}$ decay, and we could separate $\nu_{e}$ and $\nabla_{e}$ contributions from their different distributions in $y=E_{e} / E_{\nu^{*}}$

- neutrino-proton elastic scattering. This is the simplest weak neutralcurrent reaction involving hadrons. As such, the interpretation of any results is subject to some quantum chromodynamics (QCD) model dependence. Coupled with precise neutrino-electron data, this reaction can be used to test QCD assumptions. Low $-q^{2}$ neutrino-proton data can also be used to test the space-time properties of the weak neutral current.

- o-tier physics. Enormous numbers of other types of events can be collected. Here we include quasi-elastic scattcring and single-pion production. It is not clear that the vastly improved statistical sample will be useful, as systematic errors and model dependences in the interpretation are serious problems. On the other hand, the large fluxes imply that one can contemplate a relatively sma.11, heavily instrumented detector to study these processes and minimize these problems.

The group is also addressing the questions of what a detector and a neutrino facility might look like, what proton energy is optimum, and what fluxes are to be expected.

\section{MUON WORKING GROUP}

A workshop entitled "Muon Science and Facilities at Los Alamos" was held at LAMPF March 15-18, 1982 as part of the continuing series of LAMPF II planning sessions. About 45 workshop participants from the United States and abroad discussed current worldwide facilities and scientific activities using stopping muon beams with a view toward delineating future directions for such work. The workshop was organized around five working groups:

1. Particle Physics - V. W. Hughes (Yale University), Chairman;

2. Nuclear Physics - J. D. Walecka (Stanford University), Chalrman;

3. Solid-State Physics - A. Schenck (ETH Zürich), Chairman; 
4. Chemistry - D. Fleming (University of British Columbia, TRIUMF), Chairman; and

5. Facilities and Costs - J. Bradbury (Los Alamos), Chairman.

In addition, there were the following keynote speakers:

K. Nagamint (Tokyo) - "Muon Phystes Program and Facilities at the KEK-BOOM,"

$V$. W. Hughes (Yale University) - "Weak and Electromagnetic Interaction Studies with Muons," and

J. H. Brewer (University of British Columbia, TRIUMF) - "Use of Muons in the Study of Solid-State Physics and Chemistry."

The working group discussions focused on future facilities that would be possible at Los Alamos with the planned Proton Storage Ring (PSR) and LAMPF II. The PSR will produce protons with a planned maximum intensity on target of about $100 \mu \mathrm{A}$ in two pulse modes, a $1-n s-w i d e$ burst at $720 \mathrm{~Hz}$ or a 270 -ns-wide burst at $12 \mathrm{~Hz}$. Pulsed beams are important for the following types of experiments:

- studies of delayed processes where beam-associated background can be reduced by a prompt timing cut;

- experiments using intrinsically pulsed environments, such as laser pumping of muonic atoms or applications of $\mathrm{rf}$ fields;

- muonium hyperfine structure studies with line-narrowing techniques; and

- muon-spin-rotation experiments wherein all mons in a given pulse arrive at the target within a few nanoseconds, thus circumventing pile-up timing probiems.

The working model for LAMPF II was about $25 \mathrm{\mu A}$ in a dc mode at about $16-\mathrm{GeV}$ energy. The principal advantage LAMPF II would afford is a dc beam, providing an order of magnitude lower instantaneous rates than are currently achieved at LAMPF with its $6 \%$ duty factor and a much higher muon intensity than is now available at the Stopped Muon Channel (SMC).

Before the workshop a preliminary design for a superconducting-solenoidal decay beam channel was worked out by G. H. Sanders, R. Werbeck, and R. H. Heffner of Los Alamos. Table I shows the calculated values for the SMC and for the new channel at both the PSR and LAMPF II. The LAMPF II production target will be thinner than at the PSR to accommodate the increased heat production at $16 \mathrm{GeV}$.

The most striking feature of the new solenoidal decay channel is that it produces 5-10 times the muon/proton ratio compared to the SMC. Furthermore, at the PSR a relatively thick production target can be used because the proton 
energy is modest and the proton beam need not be passed to a downstream target. Thus, although the PSR has a substantially lower primary proton current, the final muon stopping rates would be about the same. The PSR, of course, provides a unique duty factor. At LAMPF II the mon yield per proton is about two orders of magnitude greater than at LAMPF/SMC because of the solenold decay channel and the increased production cross section at the higher energy. Therefore, a LAMPF II channel could produce a dc $\mu^{-}$stopping beam nearly 30 times more intense than at the SMC; the $\mu^{+}$stopping rate would be about 6 times larger. In addition to these advantages this new solenoidal channel would provide slgnificantly better rate and phase-space control.

A possible location for a muon channel coupied to the PSR was found south of the Weapons Neutron Resea:ch (WNR) facility. Layouts and designs of the proton transport line, target cell, and shielding were studied and a rough cost estimate was obtained.

One of the most important conclusions reached at the workshop regarding beam characteristics was the need for high-intensity, 1 ow-momentum ( $<50-\mathrm{MeV} / \mathrm{c}$ ) $\mu^{+}$and $\mu^{-}$beams. Consequently, future beam-line designs for either the PSR or LAMPF II w11l focus on these low-momentum beams in addition to the decay beam discussed above.

In general there was great enthusiasm generated at the workshop for the future of physics with stopping muon beams. No other particle probe at any of the world's meson facilities provides the extraordinary range of scientific study, from chemistry to particle physics, as does the muon. Equally great was the enthusiasm shown for possible new muon facilities at Los Alamos. 
TABLE I

COMPARISON OF CHANNEL FLUXES

\begin{tabular}{|c|c|c|c|c|c|c|c|}
\hline (1) & (2) & $(3)^{a}$ & (4) & $(5)^{b}$ & $(6)^{c}$ & (7) & $(8)^{d}$ \\
\hline Location & Target & $\begin{array}{c}I p \\
(\mu A)\end{array}$ & $\begin{array}{l}\Delta \mathrm{p}_{\pi} / \mathrm{p}_{\pi} \\
(\mathrm{FWHM}) \\
\end{array}$ & $\begin{array}{c}\Delta \Omega_{\pi} \\
(\mathrm{str}) \\
\end{array}$ & $\begin{array}{c}\text { W Spot } \\
\text { Size } \\
\left(\mathrm{cm}^{2}\right) \\
\end{array}$ & $\mu-/ \mu A \cdot s$ & $\mu^{-} / \mu A \cdot s \cdot q$ \\
\hline LAMPF II & $8-\mathrm{cm} \mathrm{Be}$ & 25 & 0.158 & 0.196 & 100 & $1.6 \times 10^{7}$ & $5.3 \times 10^{4}$ \\
\hline PSR & $30-\mathrm{cm} \mathrm{Be}$ & $15-100$ & 0.158 & 0.196 & 100 & $8.8 \times 10^{5}$ & $3.0 \times 10^{3}$ \\
\hline SMC & $5-\mathrm{cm} \mathrm{C}$ & 500 & 0.134 & 0.456 & 50 & $1.4 \times 10^{4}$ & $0.93 \times 10^{2}$ \\
\hline
\end{tabular}

${ }^{a}$ Column 3 contains estimates of proton intensities for the PSR and LAMPF II. The SMC value is a nominal one as of February 1982.

${ }^{\mathrm{b}}$ Column 5 is the geometrical solid angle subtended by the front aperture of the channel.

${ }^{c}$ CoJumn 6 is for the FWHM and thus contains about $50 \%$ of the beam.

${ }^{\mathrm{d}}$ Column 8 is for the $\mathrm{CH} 2$ stopping target ( $\mathrm{range}$ spread $\simeq 3 \mathrm{~g} / \mathrm{cm}^{2}$ ) and uses the nominal spot size in Column 6. As an example, consider a $1-\mathrm{cm}$-thick, $50-\mathrm{cm}^{2} \mathrm{CH}_{2}$ target:

\begin{tabular}{|c|c|c|c|}
\hline & & $\mu^{-}$stops/s & $\mu^{+}$stops/s \\
\hline LAMPF & $I I\left(\begin{array}{ll}25 & \mu A\end{array}\right)$ & $6.6 \times 10^{7}$ & $6.6 \times 10^{7}$ \\
\hline PSR & $(15 \mu A)^{a a}$ & $2.3 \times 10^{6}$ & $1.1 \times 10^{7}$ \\
\hline$S M C$ & $(500 \mu \mathrm{A})$ & $2.4 \times 10^{6}$ & $1.2 \times 10^{7}$ \\
\hline
\end{tabular}

${ }^{a}$ PSR rates corrected for proton absorption in the thick target. 


\section{Printed in the United States of America Avalable from \\ National Technical Jnformation Service US Department of Commerce 5285 Port Royal Road Springlield, VA 22161 \\ Microfiche (A01)}

NTIS

Page Range Price Code

\subsection{5} 026.050

051-075

076-100

101-125

126-150

$A 02$
$A 03$
$A 04$
A05
A06
A07

NTIS

NTIS

Price Code

Page Range Price Codc

151.175

176-200

201-225

226.250

251-275

276-300

A08
A09

A10

A1I

A12

A13

\begin{tabular}{cc} 
Page Range & $\begin{array}{c}\text { NTIS } \\
\text { Price Code }\end{array}$ \\
\hline 301.325 & A 14 \\
326.350 & A 15 \\
351.375 & A 16 \\
$376-400$ & A 17 \\
401.425 & A 18 \\
$426-450$ & A 19
\end{tabular}

Page Range Prise Code

$451-475$

476-500

$501-525$

526-550

551575

576-600

601-up"
Price Code

A20

A21

A22

A23

A24

A25

"Contact " iIS for a price quote. 


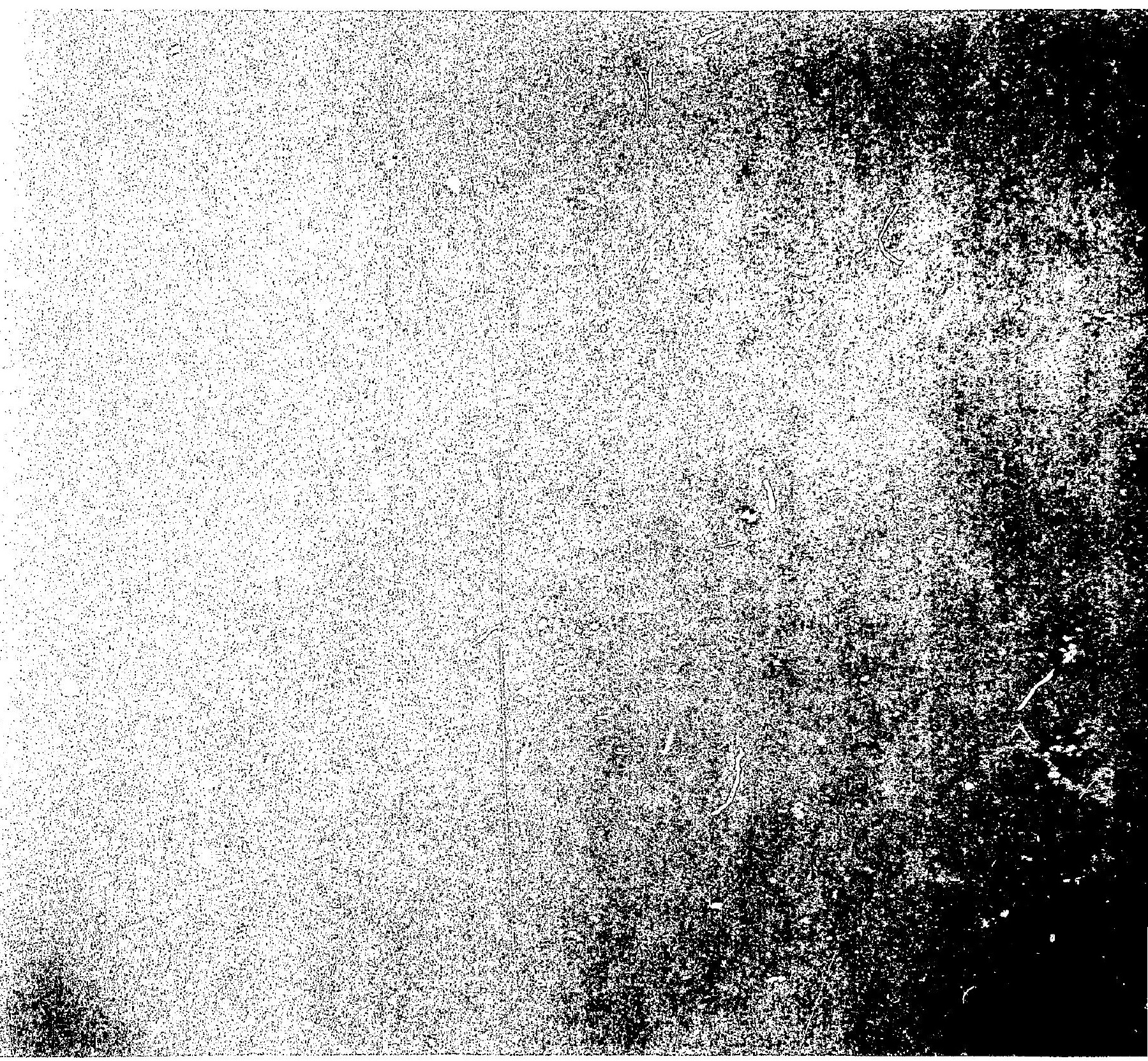

Los Alamos 